\title{
Magneto-Optics of Excitons Interacting with Magnetic lons in CdSe/CdMnS Colloidal Nanoplatelets
}

Elena V. Shornikova,* Dmitri R. Yakovlev,* Danil O. Tolmachev, Vitalii Yu. Ivanov, Ina V. Kalitukha, Victor F. Sapega, Dennis Kudlacik, Yuri G. Kusrayev, Aleksandr A. Golovatenko, Sushant Shendre, Savas Delikanli, Hilmi Volkan Demir, and Manfred Bayer

Cite This: ACS Nano 2020, 14, 9032-9041

Read Online

ACCESS $\quad$ Wlll Metrics \& More | 回 Article Recommendations

Supporting Information

ABSTRACT: Excitons in diluted magnetic semiconductors represent excellent probes for studying the magnetic properties of these materials. Various magneto-optical effects, which depend sensitively on the exchange interaction of the excitons with the localized spins of the magnetic ions can be used for probing. Here, we study core/shell $\mathrm{CdSe} /(\mathrm{Cd}, \mathrm{Mn}) \mathrm{S}$ colloidal nanoplatelets hosting diluted magnetic semiconductor layers. The inclusion of the magnetic $\mathrm{Mn}^{2+}$ ions is evidenced by three magneto-optical techniques using high magnetic fields up to 15 $T$ : polarized photoluminescence, optically detected magnetic

\section{$\mathrm{CdSe} / \mathrm{Cd}_{x} \mathrm{Mn}_{1-x} \mathrm{~S}$}

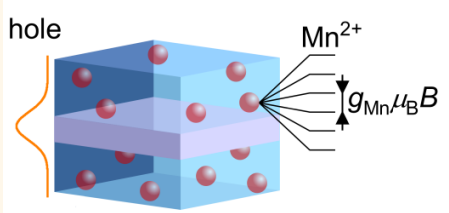

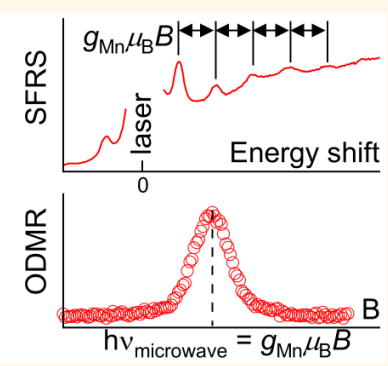
resonance, and spin-flip Raman scattering. We show that the holes in the excitons play the dominant role in exchange interaction with magnetic ions. We suggest and test an approach for evaluation of the $\mathrm{Mn}^{2+}$ concentration based on the spinlattice relaxation dynamics of the $\mathrm{Mn}^{2+}$ spin system.

KEYWORDS: diluted magnetic semiconductors, CdSe nanoplatelet, colloidal nanocrystals, magneto-optics, spin-flip Raman scattering, optically detected magnetic resonance

$I^{n}$ ncorporation of magnetic ions in colloidal nanocrystals (NCs) opens exciting opportunities for the engineering of spintronics devices. ${ }^{1-4}$ The underlying idea to exploit the strong $\mathrm{sp}-\mathrm{d}$ exchange interactions of electrons and holes with the localized spins of magnetic ions originates from the physics of diluted magnetic semiconductors (DMSs). ${ }^{5}$ This research direction was established first for bulk DMS materials and later was successfully extended for epitaxially grown DMS heterostructures, including quantum wells and quantum dots. ${ }^{6,7}$ In colloidal nanostructures, it is still at an early stage, while several important results have been already achieved. The giant Zeeman splitting was demonstrated by measuring the magnetic circular dicroism, ${ }^{8-11}$ including the photoinduced magnetism in $\mathrm{Ag}^{+}$doped $\mathrm{CdSe} \mathrm{NCs}^{12}$ and evidenced by polarized photoluminescence (PL) in external magnetic fields. ${ }^{13-18}$ The exchange interaction of excitons with $\mathrm{Mn}^{2+}$ ions was proven by optically detected magnetic resonance (ODMR). ${ }^{19}$ Magnetic polaron formation was reported, ${ }^{3,20-22}$ and the influence of $\mathrm{Mn}^{2+}$ spin fluctuations was considered. ${ }^{23}$

Magneto-optical studies of the exciton emission, its giant Zeeman splitting and polarization, are a valuable tool for investigation of DMS nanostructures. There is, however, a limitation for the parameters of DMS NCs to provide efficient exciton PL. The $\mathrm{Mn}^{2+}$ ions have an absorption band at the energy of about $2.1 \mathrm{eV}$ associated with the internal transition ${ }^{6} A_{1} \rightarrow{ }^{4} T_{1}$; the corresponding ${ }^{4} T_{1} \rightarrow{ }^{6} A_{1}$ emission is located at about $2.0 \mathrm{eV}$. This means that in $(\mathrm{Cd}, \mathrm{Mn}) \mathrm{Se} \mathrm{NCs}$, the exciton resonance should be considerably detuned from this energy, because the efficient energy transfer to the $\mathrm{Mn}^{2+}$ ions would otherwise provide a nonradiative recombination channel for the excitons, quenching their emission. For this reason, $(\mathrm{Cd}, \mathrm{Mn}) \mathrm{Se}$ spherical NCs with large diameters were synthesized in order to keep the exciton emission energy below $2.1 \mathrm{eV}$. $^{11,13,21}$

Nanoplatelets (NPLs) are an emerging class of colloidal nanocrystals, which are atomically flat with several monolayer thickness, resembling free-standing quantum wells. ${ }^{24}$ NPLs with magnetic $\mathrm{Mn}^{2+}$ ions were synthesized in $2015,{ }^{16}$ providing

Received: May 14, 2020

Accepted: June 25, 2020

Published: June 25, 2020 
remarkable opportunities for wave function engineering. ${ }^{11,25-27}$ The $\mathrm{Mn}^{2+}$ ions were incorporated in the NPL cores ${ }^{28}$ or shells. ${ }^{16,26}$

In this paper, we study the magneto-optical properties of core/shell CdSe/Cd $\mathrm{Cd}_{1-x} \mathrm{Mn}_{x} \mathrm{~S}$ NPLs, which arise from excitons interacting with the magnetic ions. Three experimental approaches are used: (i) polarized PL in external magnetic fields, (ii) optically detected magnetic resonance of the $\mathrm{Mn}^{2+}$ ions, and (iii) spin-flip Raman scattering. We measure the spinlattice relaxation dynamics of the $\mathrm{Mn}^{2+}$ spin system and suggest an approach for evaluation of the $\mathrm{Mn}^{2+}$ concentration.

Four NPL samples were grown for this study, see refs 16, 29, and 30 and Supporting Information S1 for details. All of them have a 2-monolayer thick CdSe core and 4-monolayer thick shells cladding the core. The reference sample \#0 has nonmagnetic CdS shells, and the other three DMS NPLs have $\mathrm{Cd}_{1-x} \mathrm{Mn}_{x} \mathrm{~S}$ shells with $\mathrm{Mn}^{2+}$ concentrations $x$ ranging from 0.009 to 0.029 . The sample parameters are given in Table 1 .

Table 1. Parameters of the Studied CdSe/CdS and CdSe/ $\mathrm{Cd}_{1-x} \mathrm{Mn}_{x} \mathrm{~S}$ NPLs

\begin{tabular}{ccccc} 
sample \# & $\begin{array}{c}\mathrm{Mn}^{2+} \text { content from } \\
\text { ICP-MS }\end{array}$ & $\begin{array}{c}\tau_{\mathrm{SLR}} \\
\mu \mathrm{s}\end{array}$ & $\begin{array}{c}\mathrm{Mn}^{2+} \text { content from } \\
\text { ODMR }\end{array}$ & $\begin{array}{c}\Delta E_{\mathrm{AF}} \\
\mathrm{meV}\end{array}$ \\
0 & 0 & - & - & 1.7 \\
1 & 0.012 & 405 & 0.009 & 1.6 \\
2 & 0.019 & 350 & 0.010 & 1.8 \\
3 & 0.050 & 20 & 0.029 & 1.9 \\
\hline
\end{tabular}

Note that the $\mathrm{Mn}^{2+}$ concentrations obtained by inductively coupled plasma mass spectrometry (ICP-MS) measurements differ from the values that we evaluate from the spin-lattice relaxation dynamics. We are convinced that the latter values are more reliable and we use them in the paper.

\section{RESULTS AND DISCUSSION}

Specifics of DMS Heterostructures. The band structure of the $\mathrm{CdSe} / \mathrm{Cd}_{1-x} \mathrm{Mn}_{x} \mathrm{~S}$ NPLs is shown schematically in Figure 2a. The CdSe core with zincblende lattice (Figure S1) has the bandgap energy $E_{\mathrm{g}}^{\mathrm{CdSe}}=1.75 \mathrm{eV}^{31}$ and is sandwiched between shells with $E_{\mathrm{g}}^{\mathrm{CS}}=2.50 \mathrm{eV}$. Note that the $E_{\mathrm{g}}^{\mathrm{CdSe}}=1.84 \mathrm{eV}$ used in refs 19 and 26 corresponds to the wurtzite lattice. The conduction and valence band offsets between CdSe and CdS are not precisely known. However, the valence band offsets reported in literature are large, so that the hole is believed to be confined in the CdSe core. The reported conduction band offsets range from 300 to $0 \mathrm{meV}$, and this value depends on the crystal structure, NC size, lattice strain, and temperature. Due to the quite weak, if present at all, confinement, the electron wave function leaks into the $\mathrm{CdS}$ shell (for references, see ref 32 ). The electron and hole wave functions are centered in the nonmagnetic CdSe core and only partially penetrate into the DMS shell. For this reason, all exchange effects in the studied DMS NPLs are expected to be reduced compared to bulk DMSs with the same $\mathrm{Mn}^{2+}$ concentration. Quantum mechanical calculations give an estimate of the electron wave function fraction in the shell about $60 \%$ and the hole fraction about $30 \%$, see Supporting Information S5.

There are several factors that need to be taken into account for evaluation of the strength of the exciton and carrier exchange interactions with the $\mathrm{Mn}^{2+}$ spins in CdSe/Cd ${ }_{1-x} \mathrm{Mn}_{x} \mathrm{~S}$ NPLs:

(i) Penetration of the electron and hole wave functions into the DMS shells. Note that in bulk II-VI DMSs, the exchange interaction of holes is $4-8$ times stronger than that of the electrons. 6,7 Correspondingly, the holes provide the dominating contributions to the magnetooptical effects, like the giant Zeeman splitting of exciton states, Faraday rotation, formation of exciton magnetic polarons, etc.

(ii) Modification of the electron exchange constant $\alpha$ by strong quantization. $\alpha$ is reduced with increasing confinement and can even change its sign. ${ }^{33,34}$

(iii) Variations of the magnetic properties of the $\mathrm{Mn}^{2+}$ spin system, which are controlled by the $\mathrm{Mn}^{2+}-\mathrm{Mn}^{2+}$ interactions and are different in bulk DMSs and in thin DMS layers or at the interfaces between DMS and nonmagnetic layers, because of different statistics of neighboring $\mathrm{Mn}^{2+}$ spins. ${ }^{35,36}$

Therefore, it is a challenging task to account properly for the contributions of these factors to the magneto-optical properties of the studied NPLs. As a result, one can not use most of the established approaches in DMS physics for evaluation of the $\mathrm{Mn}^{2+}$ content by means of magneto-optical techniques.

Time-Integrated and Time-Resolved Photoluminescence. Figure la shows PL spectra of $\mathrm{CdSe} / \mathrm{CdS}$ (sample \#0) and $\mathrm{CdSe} / \mathrm{Cd}_{0.991} \mathrm{Mn}_{0.009} \mathrm{~S}$ (sample \#1) NPLs. Both spectra are very similar to each other, so that implementation of a small $\mathrm{Mn}^{2+}$ concentration does not change the PL. The emission lines of both samples are centered at $2.127 \mathrm{eV}$ (red arrow) and have
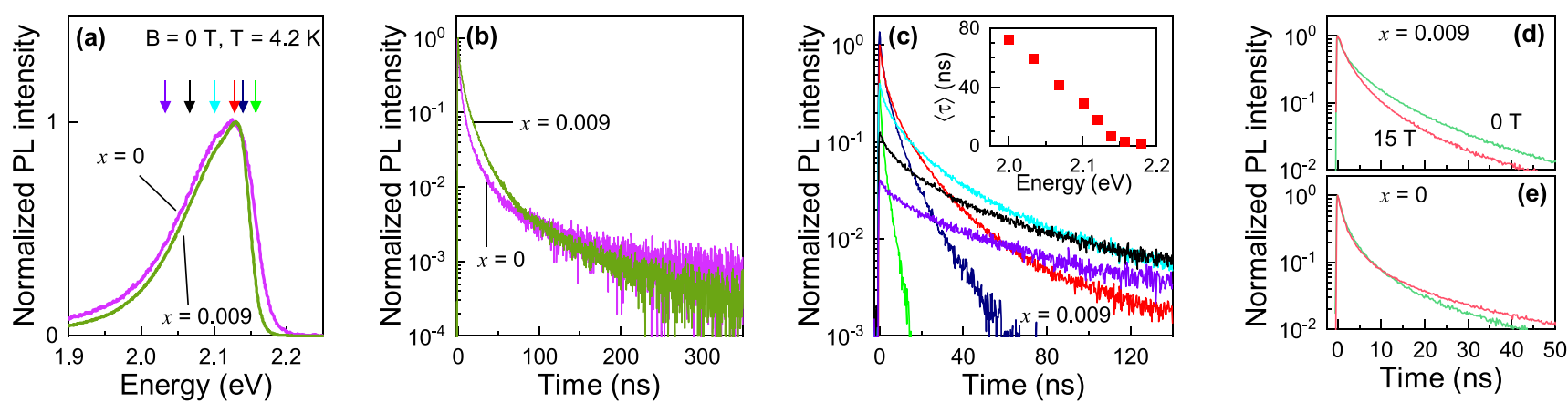

Figure 1. (a) PL spectra of CdSe/CdS (sample \#0, pink) and CdSe/Cd ${ }_{0.991} \mathrm{Mn}_{0.009} S$ (sample \#1, green) NPLs in B= $0 \mathrm{~T}$. (b) PL decay traces of sample \#0 (pink) and sample \#1 (green) NPLs, measured at their PL maxima of $2.127 \mathrm{eV}$. (c) PL decay curves of CdSe/Cd $\mathrm{Cd}_{0.991} \mathrm{Mn}_{0.009} S \mathrm{NPLs}$ (sample \#1) at various detection energies shown by the arrows in panel (a) with the same color code. Inset: Spectral dependence of the average decay time $\langle\tau\rangle$ in sample \#1. (d,e) PL decay at $2.127 \mathrm{eV}$ measured in magnetic fields B= $0 \mathrm{~T}$ (green) and $15 \mathrm{~T}$ (red) in samples \#1 and \#0. All measurements performed at $T=4.2 \mathrm{~K}$. 
(a)

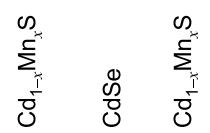

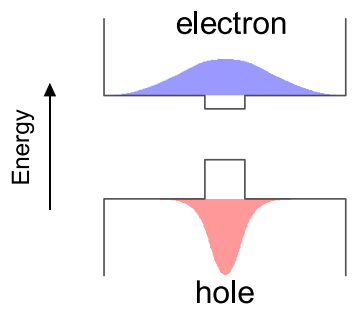

(b)

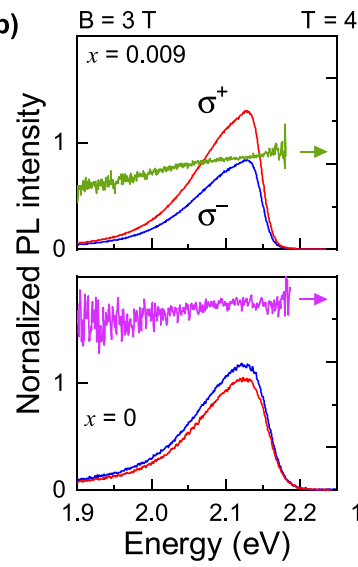

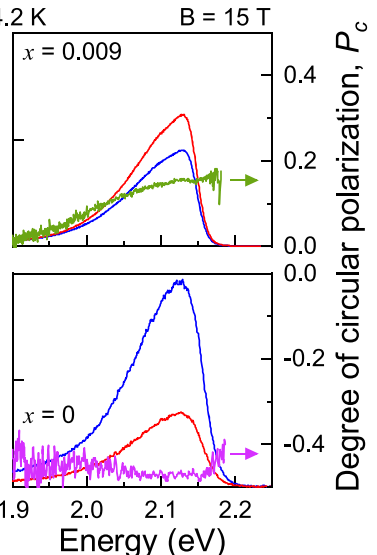

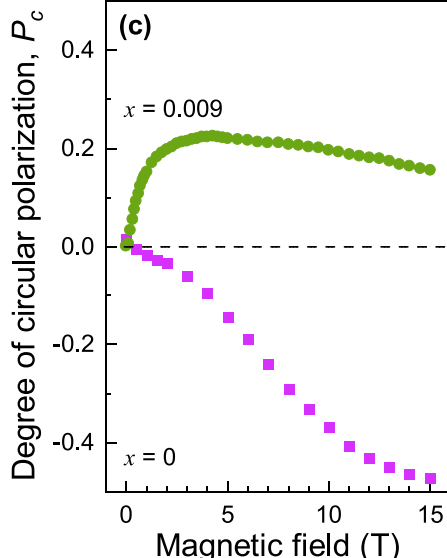

Figure 2. (a) Schematic diagram of the electron and hole wave functions (blue and red contours) in CdSe/Cd ${ }_{1-x} \mathrm{Mn}_{x} \mathrm{~S}$ NPLs for the conduction valence band offset $0.15 \mathrm{eV}$. (b) PL spectra of the $\sigma^{+}$(red) and $\sigma^{-}$(blue) polarized components in CdSe/CdS NPLs (sample \#0, bottom) and $\mathrm{CdSe} / \mathrm{Cd}_{0.991} \mathrm{Mn}_{0.009} \mathrm{~S}$ NPLs (sample \#1, top) at $T=4.2 \mathrm{~K}, B=3 \mathrm{~T}$ (left), and $15 \mathrm{~T}$ (right). (c) Magnetic field dependence of the DCP in sample \#0 (pink) and sample \#1 (green).

full widths at half-maximum of about $100 \mathrm{meV}$, which is typical for core/shell NPLs. ${ }^{37}$ As the PL is close to the ${ }^{4} T_{1} \rightarrow{ }^{6} A_{1}$ transition of the $\mathrm{Mn}^{2+}$ ions at $2.1 \mathrm{eV}$, our first task is to identify the origin of the emission from CdSe/Cd $\mathrm{Cd}_{0.991} \mathrm{Mn}_{0.009} \mathrm{~S}$ NPLs and to prove that it is dominated by exciton recombination. The similarity of the PL spectra of nonmagnetic and DMS NPLs gives a first hint for that.

The recombination dynamics can be also used for the identification of the origin of the emission. It is known that at liquid helium temperatures, the decay of the $\mathrm{Mn}^{2+}$ emission via the ${ }^{4} T_{1} \rightarrow{ }^{6} A_{1}$ transition is very slow, occurring on time scales in the $10-500 \mu \mathrm{s}$ range in bulk DMSs, ${ }^{38,39}$ like (Cd,Mn)Te, $(\mathrm{Zn}, \mathrm{Mn}) \mathrm{Te}$, and $(\mathrm{Zn}, \mathrm{Mn}) \mathrm{S}$, and is $270 \mu \mathrm{s}$ in (Cd,Mn)Se colloidal quantum dots. ${ }^{40}$ The exciton recombination dynamics is by a few orders of magnitude faster, happening for neutral excitons in the range of $1 \mathrm{~ns}$ to $1 \mu \mathrm{s}$, depending on the relative involvement of bright and dark exciton states or of a few nanoseconds for charged excitons (trions). ${ }^{37,41,42}$

The time-resolved recombination dynamics measured at the PL maxima in samples \#0 and \#1 are shown in Figure 1b. In both cases, the decay of the PL intensity takes place within $300 \mathrm{~ns}$ being 3 orders of magnitude faster than the recombination dynamics of $\mathrm{Mn}^{2+}$ ions. This allows us to conclude that the dominating part of the emission in $\mathrm{CdSe} / \mathrm{Cd}_{0.991} \mathrm{Mn}_{0.009} \mathrm{~S}$ NPLs is provided by exciton recombination and the $\mathrm{Mn}^{2+}$ emission is very weak, if present at all. The two other DMS samples have similar properties.

As it is common for the colloidal nanocrystals, the recombination dynamics in the studied NPLs do not show a monoexponential decay. For example, the decays at the PL maxima, shown in Figure $1 \mathrm{~b}$, require a fit with a three-term exponential function for a good description: $I(t)=A_{1} \exp (-t /$ $\left.\tau_{1}\right)+A_{2} \exp \left(-t / \tau_{2}\right)+A_{3} \exp \left(-t / \tau_{3}\right)$. The three decay times are $\tau_{1}=3 \mathrm{~ns}, \tau_{2}=11 \mathrm{~ns}$, and $\tau_{3}=56 \mathrm{~ns}$ for sample \#0 and $\tau_{1}=3 \mathrm{~ns}$, $\tau_{2}=12 \mathrm{~ns}$ and $\tau_{3}=45 \mathrm{~ns}$ for sample \#1. Note that they are close to each other in these nonmagnetic and DMS NPLs.

The spectral dependence of the PL dynamics in CdSe/ $\mathrm{Cd}_{0.991} \mathrm{Mn}_{0.009} \mathrm{~S}$ NPLs is given in Figure 1c. The general trend is that the decay times increase with decreasing emission energy. This is clearly seen in the inset of Figure 1c, where the spectral dependence of the average decay time $\langle\tau\rangle$ is given. $\langle\tau\rangle$ is calculated as $\langle\tau\rangle=\tau_{1} \nu_{1}+\tau_{2} \nu_{2}+\tau_{3} \nu_{3}$, where $\nu_{i}=A_{i} \tau_{i} /\left(A_{1} \tau_{1}+\right.$ $\left.A_{2} \tau_{2}+A_{3} \tau_{3}\right)$. The average decay time increases from 2 up to 70 ns from the high- to the low-energy tail. The nonmagnetic sample demonstrates the similar spectral dependence (Figure S2), which proves that it is not due to the $\mathrm{Mn}^{2+}$ emission. Such behavior is typical for ensembles of colloidal nanocrystals with an efficient Förster energy transfer. ${ }^{43,44}$

Further more, the recombination dynamics are weakly affected by external magnetic fields. This is shown in Figure $1 \mathrm{~d}, \mathrm{e}$, where the PL decays at the emission maximum are compared for $B=0$ and $15 \mathrm{~T}$.

Note that the character of the recombination dynamics at low temperatures in colloidal NPLs and its dependence on magnetic field allows one to identify whether the emission is contributed by neutral or by charged excitons. ${ }^{37,41,42}$ For example, at $T=4.2$ $\mathrm{K}$, the trion emission in CdSe/CdS NPLs with thick shells is monoexponential with a decay time of $3 \mathrm{~ns}$ and is independent of magnetic field. Contrary to that, the decay of neutral excitons in CdSe NPLs has a biexponential decay with a very fast initial component of 20 ps and a long component of $80 \mathrm{~ns}$, which shortens with increasing magnetic field.

The recombination dynamics in the NPLs studied in this paper do not clearly correspond to either neutral or charged exciton behavior, but are rather superpositions of both. Additionally, for resonant excitation, we clearly observe emission from dark excitons (Figure 4a). The bright-dark exciton energy splitting, $\Delta E_{\mathrm{AF}}$, ranges between 1.6 and $1.9 \mathrm{meV}$ (see below). Therefore, at $T=4.2 \mathrm{~K}$, the bright state has about $1 \%$ population in thermal equilibrium and should contribute to the emission. We also detect electron spin flips, which means that some of the NPLs are negatively charged, that is, they may contain negatively charged excitons (Figure 4a). From all these findings, we conclude that the PL is contributed by a recombination of neutral (bright and dark) and charged excitons. More details are given in the Supporting Information S4.

Polarized Photoluminescence in the Magnetic Field. This technique, which exploits the exciton (trion) spin polarization on their Zeeman split sublevels, is a sensitive tool to measure small spin splittings comparable with the thermal energy $k_{\mathrm{B}} T$, where $k_{\mathrm{B}}$ is the Boltzmann constant. ${ }^{37,45,46}$ The experiment is relatively easy in realization, but requires liquid helium temperatures and strong magnetic fields of 10-15 T or even up to 30-65 $\mathrm{T}$ to gather sufficient information on the linear dependence of the circular polarization degree of $\mathrm{PL}, P_{c}(B)$, on 

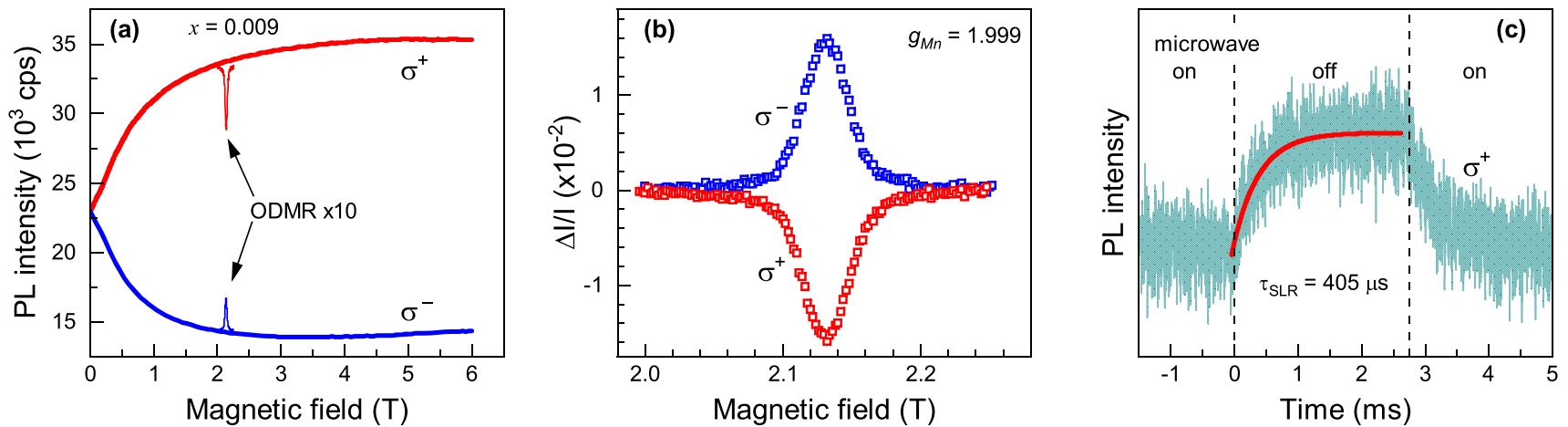

(d)
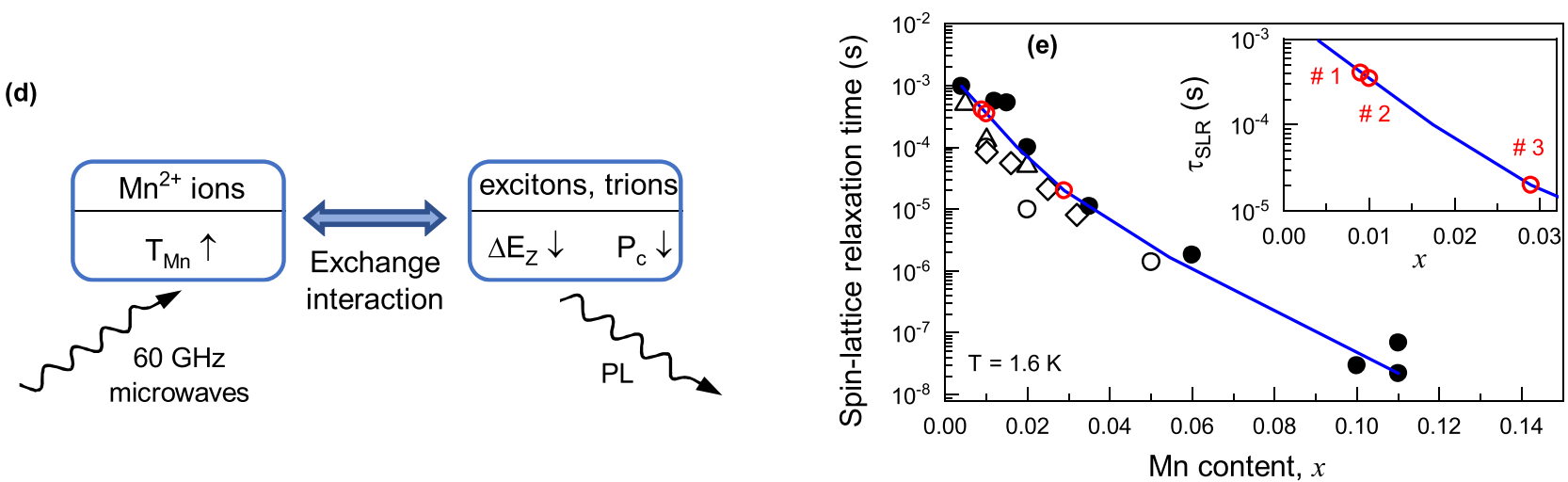

Figure 3. (a) Dependence of the $\sigma^{+}$(red) and $\sigma^{-}$(blue) circularly polarized components of PL on magnetic field in Faraday geometry for CdSe/ $\mathrm{Cd}_{0.991} \mathrm{Mn}_{0.009} \mathrm{~S}$ NPLs (sample \#1). $P_{\text {exc }}=4 \mathrm{~W} / \mathrm{cm}^{2}$ and $T=1.8 \mathrm{~K}$. The peaks show the PL intensities for application of microwaves $($ ODMR signals). Their intensities are multiplied by a factor of 10. (b) Normalized ODMR signals for the two circularly polarized components of the PL. The ODMR resonance has a line width of $\Delta B \approx 40 \mathrm{mT}$ and is located at $B=2.130 \mathrm{~T}$, which corresponds to a $g$-factor of 1.999 . The $\sigma^{+}$component decreases at resonance conditions (red), while the $\sigma^{-}$component increases (blue). (c) Temporal evolution of the $\sigma^{+}$PL component after switching on $/$ off the microwaves for resonance excitation at $B=2.130 \mathrm{~T}$. The red line is an exponential fit with a time constant $\tau_{\mathrm{SLR}}=405 \mu \mathrm{s}$. $(\mathrm{d})$ Schematic diagram of the interactions between the $\mathrm{Mn}^{2+}$ and exciton (trion) spin systems. (e) Spin-lattice relaxation time as a function of the $\mathrm{Mn}^{2+}$ content $x$; adapted from ref 35 . Blue line is a guide for the eye, and red circles are experimental data measured in the present work (see Table 1).

magnetic fields in weak fields, until it reaches saturation in high magnetic fields, $P_{c}^{\text {sat }}$. The degree of circular polarization (DCP) is defined as $P_{\mathrm{c}}=\left(I^{+}-I^{-}\right) /\left(I^{+}+I^{-}\right)$, where $I^{+}$and $I^{-}$are the PL intensities of the $\sigma^{+}$and $\sigma^{-}$circularly polarized components, respectively. The magnetic field is applied in the Faraday geometry, that is, parallel to the emission wave vector direction.

Figure $2 \mathrm{~b}$ shows polarized PL spectra of the nonmagnetic sample \#0 (bottom) and Mn-doped sample \#1 (top), measured in magnetic fields $B=3$ and $15 \mathrm{~T}$. One can clearly see the difference between DCP in nonmagnetic and DMS NPLs. First, they have opposite signs. In CdSe/CdS NPLs $P_{c}<0$, the absolute value increases with growing magnetic field about monotonically and saturates above $12 \mathrm{~T}$ (Figure $2 \mathrm{c}$ ). At $B=15$ $\mathrm{T}$, it reaches $P_{\mathrm{c}}=-0.47$. This behavior is similar to what was reported for thick-shell CdSe/CdS NPLs (see Figure 3c in ref 37 ), where the emission was provided by negatively charged excitons.

In the DMS sample $P_{c}>0$, it increases fast, reaching a plateau value of +0.22 at $B=4 \mathrm{~T}$, and then slowly decreases in higher magnetic fields. The $P_{\mathrm{c}}$ sign reversal in II-VI DMS materials, compared to the nonmagnetic reference, is clear evidence of the exchange interaction of charge carriers with the $\mathrm{Mn}^{2+}$ ions. It is provided by the signs of the exchange constants in the conduction $(\alpha>0)$ and valence bands $(\beta<0) .^{7}$ More details are given in the Supporting Information S6.

Analysis of Polarized Photoluminescence. The exciton and trion DCP can be written as

$$
P_{\mathrm{c}}(B)=-P_{\mathrm{c}}^{\mathrm{sat}} \frac{\tau}{\tau+\tau_{\mathrm{s}}} \tanh \frac{\Delta E_{\mathrm{Z}}(B)}{2 k_{\mathrm{B}} T}
$$

where $\Delta E_{\mathrm{Z}}(B)$ is the Zeeman splitting, $\tau$ is the lifetime, $\tau_{\mathrm{s}}$ is the spin relaxation time, and $P_{c}^{\text {sat }}$ is the saturation degree of polarization, which depends on the specifics of the spin level structure and NPL orientation in the ensemble.

In nonmagnetic samples, the intrinsic exciton Zeeman splitting is

$$
\Delta E_{\mathrm{Z}, \mathrm{X}}(B)=g_{\mathrm{X}} \mu_{\mathrm{B}} B
$$

where $g_{\mathrm{X}}$ is the exciton $g$-factor and $\mu_{\mathrm{B}}$ is the Bohr magneton. Accounting for the specifics of the bright and dark excitons is considered in Supporting Information S6.

In DMS samples, an additional term, $E_{\text {exch, } \mathrm{X}}(B)$, describing the exciton exchange interaction with the $\mathrm{Mn}^{2+}$ spins has to be added

$$
\Delta E_{\mathrm{Z}, \mathrm{X}}(B)=g_{\mathrm{X}} \mu_{\mathrm{B}} B+E_{\mathrm{exch}, \mathrm{X}}(B)
$$

Note that $E_{\text {exch, } \mathrm{X}}(B)$ is controlled by the exchange interaction of both electron and hole composing the exciton with the $\mathrm{Mn}^{2+}$ ions and therefore depends on the overlap of the electron and hole wave functions with the $(\mathrm{Cd}, \mathrm{Mn}) \mathrm{S}$ shells.

For the negative trion, being composed of two electrons and one hole, the Zeeman splitting is determined by the hole splitting: 


$$
\Delta E_{\mathrm{Z}, \mathrm{h}}(B)=-3 g_{\mathrm{h}} \mu_{\mathrm{B}} B
$$

where $g_{\mathrm{h}}$ is the hole $g$-factor. In DMS samples

$$
\Delta E_{\mathrm{Z}, \mathrm{h}}(B)=-3 g_{\mathrm{h}} \mu_{\mathrm{B}} B+E_{\text {exch, } \mathrm{h}}(B)
$$

$E_{\text {exch,h }}(B)$ is determined by the exchange interaction of the hole with the $\mathrm{Mn}^{2+}$ ions. Here we use the definition of the hole $g$ factor sign that is commonly used for colloidal nanocrystals. ${ }^{37,47}$ In the frame of this convention, the intrinsic hole Zeeman splitting provided by the negative hole $g$-factor $\left(g_{\mathrm{h}}<0\right)$ is in competition with the hole exchange splitting determined by $\beta<$ 0 . On the other hand, for the conduction band electron, both the intrinsic Zeeman splitting $\left(g_{\mathrm{e}}>0\right)$ and the exchange one with $\alpha>0$ add to each other.

The electron $g$-factor is $g_{\mathrm{e}}=+1.70$ in CdSe/CdS NPLs (see below). The hole $g$-factor $g_{\mathrm{h}}=-0.7$ was measured in high magnetic fields. ${ }^{37}$ For small $\Delta E_{\mathrm{AF}}$, as in our case, the $g$-factor of the bright exciton is $g_{\mathrm{XA}}=-g_{\mathrm{e}}-3 g_{\mathrm{h}}=+0.4$. This value matches well with $g_{\mathrm{XA}}=+0.32$ measured in the 4-monolayer thick bare core NPLs by absorption spectroscopy in high magnetic fields. ${ }^{48}$ For the dark exciton, $g_{\mathrm{XF}}=g_{\mathrm{e}}-3 g_{\mathrm{h}}=+3.8$. One can see from eq 1 that the negative DCP found in experiment requires $g_{\mathrm{X}}>0$, that is, can be achieved by the bright and the dark excitons. In case of the negative trion, $P_{\mathrm{c}}<0$ requires $g_{\mathrm{h}}<0$, see eqs 1 and 4 , which is indeed the case for $\mathrm{CdSe} / \mathrm{CdS}$ NPLs. To summarize, the negative DCP observed in nonmagnetic CdSe/CdS NPLs can be provided by the dark and bright excitons and the negative trions.

In DMS NPLs, the polarization is positive, which requires a negative sign of the Zeeman splitting $\Delta E_{\mathrm{Z}}$. One can see from eqs 3 and 5 that this can be the case when the intrinsic and exchange terms have different signs as well as when for the exciton case $\left|E_{\text {exch, } \mathrm{X}}(B)\right|>\left|g_{\mathrm{X}} \mu_{\mathrm{B}} B\right|$ and the trion case $\left|E_{\text {exch,h }}(B)\right|>\left|3 g_{\mathrm{h}} \mu_{\mathrm{B}} B\right|$. In the trion case, the fulfillment of this condition is solely provided by the hole exchange interaction with the $\mathrm{Mn}^{2+}$ ions, that is, requires a sufficiently large penetration of the hole wave function into the DMS shells. In CdSe, $g_{\mathrm{e}}$ and $\alpha$ are both positive, and therefore, the Zeeman splitting for the conduction band electron can not be inverted. As a result, for the exciton case, the inversion of the DCP sign can also be provided only by the hole exchange with the $\mathrm{Mn}^{2+}$ spins. To support this conclusion, we provide in the Supporting Information S6 results of model calculations for the bright and dark excitons and for the negative trions for various penetrations of the hole wave functions into the DMS shells.

Optically Detected Magnetic Resonance. The ODMR technique combines the advantage of resonant excitation of spin states by microwave radiation with the high sensitivity of optical detection of the induced changes. It is especially useful for the investigation of semiconductor nanostructures, whose small volume is not sufficient to provide sufficiently strong signals for the electron paramagnetic resonance technique. Additionally, the possibility of spectrally selective detection on specific optical resonances, for example, the exciton or impurity related emission, allows one to obtain a clear identification of the addressed electronic transitions.

In the case of diluted magnetic semiconductors, the resonant microwave heating of the $\mathrm{Mn}^{2+}$ ions increases the $\mathrm{Mn}^{2+}$ spin temperature $T_{\mathrm{Mn}}$ and, consequently, reduces the $\mathrm{Mn}^{2+}$ spin polarization $\left\langle S_{\mathrm{Mn}}\right\rangle$. These changes can be detected optically via the excitons or trions interacting with the $\mathrm{Mn}^{2+}$ spins, see Figure $3 \mathrm{~d}$. The application of the ODMR technique to quantum well structures based on (Zn,Mn)Se DMSs is discussed in refs 35, 49, and 50. There it was shown that the resonant heating of the $\mathrm{Mn}^{2+}$ spin system can be detected by several effects: (i) the decrease of the exciton giant Zeeman splitting, resulting in a spectral shift of the exciton emission line, (ii) the decrease of the circular polarization degree induced by the magnetic field, and (iii) the redistribution of the emission intensity between the exciton line and the $\mathrm{Mn}^{2+}$ emission band. Recently, ODMR measured at $10 \mathrm{GHz}$ microwave radiation via polarized $\mathrm{PL}$ was reported for $\mathrm{CdSe} / \mathrm{Cd}_{1-x} \mathrm{Mn}_{x} \mathrm{~S}$ NPLs. ${ }^{19}$

Figure 3a shows the intensities of the $\sigma^{+}$and $\sigma^{-}$PL components of the $\mathrm{CdSe} / \mathrm{Cd}_{0.991} \mathrm{Mn}_{0.009} \mathrm{~S}$ NPLs (sample \#1), measured versus magnetic field without and with microwaves. As discussed above, the $\sigma^{+}$component has a higher intensity due to the stronger thermal population of the excitons (trions) on the associated Zeeman sublevels split in magnetic field. Without microwaves, the intensities of these components change smoothly with magnetic field, following the DCP trend shown in Figure $2 \mathrm{c}$. In the presence of $59.6 \mathrm{GHz}$ microwave radiation, two sharp resonances are observed at $B=2.130 \mathrm{~T}$. The resonant decrease of the $\sigma^{+}$intensity and the correlated increase of the $\sigma^{-}$ intensity evidence heating of the $\mathrm{Mn}^{2+}$ spin system, which accordingly decreases the exciton (trion) giant Zeeman splitting and the exciton (trion) DCP. ${ }^{35,50}$ The PL intensity variations normalized to the relative PL intensities without microwaves (I) are shown in more detail in Figure $3 \mathrm{~b}$. They represent broad peaks with a width of $\Delta B=40 \mathrm{mT}$ and are centered at $B=2.130$ $\mathrm{T}$ corresponding to a $g$-factor of $1.999 \pm 0.005$. This $g$-factor matches with the $\mathrm{Mn}^{2+}$ value of $g_{\mathrm{Mn}}=2.01$, reported for $\mathrm{ZnS}: \mathrm{Mn}^{2+}$ and CdTe: $\mathrm{Mn}^{2+}$ in electron spin resonance measurements. $^{51,52}$

The spin-lattice relaxation (SLR) dynamics of the $\mathrm{Mn}^{2+}$ spin system can be measured by modulating the microwave radiation between on and off and time-resolved detection of the changes induced thereby, reflecting cooling or heating of the $\mathrm{Mn}^{2+}$ spins. An example of such a measurement for CdSe $/ \mathrm{Cd}_{0.991} \mathrm{Mn}_{0.009} \mathrm{~S}$ NPLs is shown in Figure 3c. Here, the red line is an exponential fit with the characteristic spin-lattice relaxation time $\tau_{\text {SLR }}=405$ $\mu$ s. Similar measurements performed for samples \#2 and \#3 give 350 and $20 \mu$ s, respectively (Table 1 ).

As we discussed above, most of the magneto-optical approaches that are commonly used for evaluation of the $\mathrm{Mn}^{2+}$ concentration in bulk DMSs cannot be directly applied to $\mathrm{CdSe} / \mathrm{Cd}_{1-x} \mathrm{Mn}_{x} \mathrm{~S}$ NPLs. We suggest that a quite accurate evaluation can be achieved from the spin-lattice relaxation time $\tau_{\mathrm{SLR}}$. It is known that the $\tau_{\mathrm{SLR}}$ of the $\mathrm{Mn}^{2+}$ ions in II-VI semiconductors has a very strong dependence on the $\mathrm{Mn}^{2+}$ concentration, which covers about 5 orders of magnitude from 1 ms down to 10 ns with increasing $x$ from 0.004 up to 0.11 , see Figure $3 \mathrm{e}$, where the data from Figure 8.10 in ref 35 are reproduced. This strong dependence arises from the quenching of the orbital momentum of the d-electrons in the $\mathrm{Mn}^{2+}$ ion, that is, it has zero orbital momentum $(L=0)$ and does not interact with the phonon system. The only mechanisms that provide spin-lattice relaxation for the $\mathrm{Mn}^{2+}$ ions are given by the $\mathrm{Mn}^{2+}-$ $\mathrm{Mn}^{2+}$ interactions, which obviously are strongly dependent on the number of neighboring $\mathrm{Mn}^{2+}$ ions and the distances between them, which in turn strongly change with increasing $\mathrm{Mn}^{2+}$ concentration. The red circles in Figure $3 \mathrm{e}$ mark the times that we measured for the $\mathrm{CdSe} / \mathrm{Cd}_{1-x} \mathrm{Mn}_{x} \mathrm{~S}$ NPLs. Their comparison with the literature data shown by the symbols allows us to evaluate the $\mathrm{Mn}^{2+}$ concentration for the studied samples. As one can see from Table 1 , the $\mathrm{Mn}^{2+}$ concentration measured by the ICP-MS method is in good agreement with our data for 

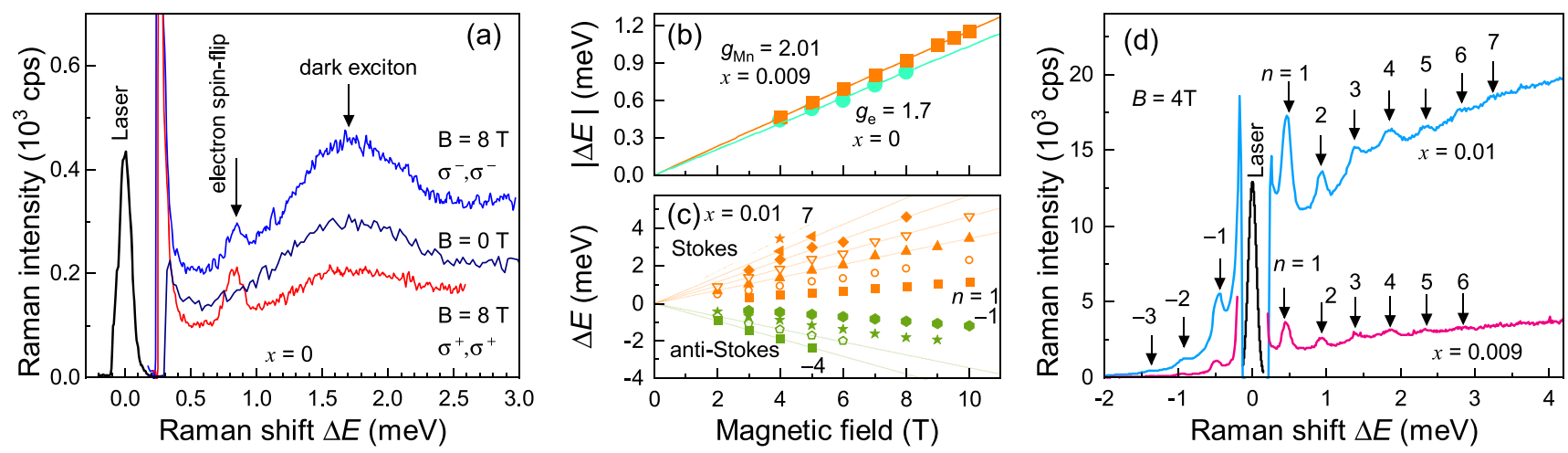

Figure 4. (a) Raman spectrum for the circularly copolarized configuration in Faraday geometry for the nonmagnetic CdSe/CdS NPLs (sample $\# 0) . E_{\text {exc }}=2.165 \mathrm{eV}, P_{\text {exc }}=0.2 \mathrm{~W} / \mathrm{cm}^{2}, T=1.6 \mathrm{~K}, B=0$ and $8 \mathrm{~T}$. (b) Magnetic field dependence of the absolute Stokes energy shifts of the $n=1$ $\mathrm{Mn}^{2+}$ resonance in CdSe/ $\mathrm{Cd}_{0.991} \mathrm{Mn}_{0.009} \mathrm{~S}$ (sample \#1, orange squares) and of the electron spin flip in CdSe/CdS NPLs (sample \#0, green circles) in Faraday geometry. Lines are linear fits to the data. (c) Magnetic field dependence of the relative Raman shifts of all detectable Mn ${ }^{2+}$ resonances in CdSe $/ \mathrm{Cd}_{0.99} \mathrm{Mn}_{0.01} \mathrm{~S}$ NPLs in Voigt geometry. (d) $\mathrm{Mn}^{2+}$ spin scattering spectra for the copolarized configuration in Faraday geometry for the CdSe $/ \mathrm{Cd}_{0.991} \mathrm{Mn}_{0.009} \mathrm{~S}$ (sample \#1, pink) and CdSe $/ \mathrm{Cd}_{0.99} \mathrm{Mn}_{0.01} \mathrm{~S}(\mathrm{sample} \# 2$, blue $) \mathrm{NPLs} . E_{\text {exc }}=2.151 \mathrm{eV}, P_{\text {exc }}=1.6 \mathrm{~W} / \mathrm{cm}^{2}, B$ $=4 \mathrm{~T}, \mathrm{~T}=1.6 \mathrm{~K}$. In Faraday geometry up to 7 (3) $\mathrm{Mn}^{2+}$ resonances are observed in the Stokes (anti-Stokes) spectral ranges.

samples \#1 and \#2, but differs for sample \#3. We emphasize that the suggested approach for evaluation of the $\mathrm{Mn}^{2+}$ concentration is very reliable and could be widely used for nanostructures. Note that in layers with a few monolayer thickness, $\mathrm{Mn}^{2+}$ ions have less $\mathrm{Mn}^{2+}$ neighbors than in bulk, which results in longer SLR dynamics. We studied this effect in (Cd,Mn)Te digital alloys grown by molecular beam epitaxy. ${ }^{36} \mathrm{We}$ found that in the layers with a thickness of 3 monolayers and larger, the SLR times are the same as in bulk, but in 1 monolayer thick layers, the time can be longer by a factor of 5 , which corresponds to a factor 2 underestimation of the $\mathrm{Mn}^{2+}$ concentration.

Spin-Flip Raman Scattering. The SFRS spectroscopy is a sophisticated tool to investigate the Zeeman splittings of carriers (electrons or holes), excitons, or magnetic ions. It provides detailed information about their spin structure and spin interactions. The polarization properties of the SFRS lines deliver information on the symmetries of the involved states and allow one to identify the responsible flip mechanisms. In SFRS, the Zeeman splitting is obtained from the Raman shift, which is equal to the energy shift between the laser photon energy and the energy of the scattered light. The technique was successfully used for investigation of the exchange interactions of carriers and excitons with the magnetic ions in DMS bulk samples ${ }^{6,53}$ and quantum well structures. ${ }^{54-56} \mathrm{We}$ showed recently that nonmagnetic $\mathrm{CdSe}$ and $\mathrm{CdSe} / \mathrm{CdS}$ NPLs can be studied by SFRS, ${ }^{37,57}$ but this technique had not been used so far for DMS colloidal nanocrystals.

Figure $4 \mathrm{a}$ shows Raman spectra of the CdSe/CdS NPLs (sample \#0) for resonant excitation of the exciton state at $E_{\text {exc }}=$ $2.165 \mathrm{eV}$. Here positive values of the Raman shift $\Delta E$ correspond to a Stokes shift of the scattered photons to lower energies. At zero magnetic field, there is a relatively broad line with a full width at half-maximum of $0.5 \mathrm{meV}$, whose maximum is shifted by $1.7 \mathrm{meV}$. This shift does not change in the applied magnetic field of $B=8 \mathrm{~T}$. We assign it to the energy splitting between the bright and dark exciton states $\Delta E_{\mathrm{AF}} \cdot{ }^{4 \mathrm{P}}$ The dark exciton line was observed in all studied samples with $\Delta E_{\mathrm{AF}}$ ranging between 1.6 and $1.9 \mathrm{meV}$ (Table 1 ). This supports our assumption that the dark excitons contribute to the emission from the $\mathrm{CdSe} / \mathrm{Cd}_{1-x} \mathrm{Mn}_{x} \mathrm{~S}$ NPLs.

At $B=8 \mathrm{~T}$, a narrow line associated with the electron spin-flip, shifted by $0.82 \mathrm{meV}$ from the laser, is seen in the Raman spectrum (Figure 4a). Its shift depends linearly on magnetic field (Figure $4 \mathrm{~b}$, green circles). A fit with $|\Delta E|=\left|g_{\mathrm{e}}\right| \mu_{\mathrm{B}} B$ gives the $g$ factor value $\left|g_{\mathrm{e}}\right|=1.70 \pm 0.02$, which is close to the electron $g$ factor measured in CdSe and CdSe/CdS NPLs. ${ }^{37,57,58}$ Note that $g_{\mathrm{e}}>0$ in bulk CdSe and in these structures.

Figure $4 \mathrm{~d}$ shows Raman spectra of $\mathrm{CdSe} / \mathrm{Cd}_{0.991} \mathrm{Mn}_{0.009} \mathrm{~S}$ and $\mathrm{CdSe} / \mathrm{Cd}_{0.99} \mathrm{Mn}_{0.01} \mathrm{~S}$ NPLs measured at $B=4 \mathrm{~T}$ with resonant excitation of the exciton at $E_{\mathrm{exc}}=2.151 \mathrm{eV}, T=1.6 \mathrm{~K}$. They are obviously very different compared to the spectra in Figure $4 \mathrm{a}$. No electron spin-flip is detected; instead, a set of equidistant lines is observed. Up to seven lines in the Stokes and up to three lines in the anti-Stokes energy range can be resolved. All these lines shift linearly with magnetic field (see Figure 4c), following the equation $\Delta E=n g_{\mathrm{Mn}} \mu_{\mathrm{B}} B$, where $n$ is an integer number. An accurate evaluation of $g_{\mathrm{Mn}}=2.01 \pm 0.03$ is obtained from the fit of the line with $n=1$, shown in Figure $4 \mathrm{~b}$ by orange squares. This $g$-factor matches well with the $\mathrm{Mn}^{2+} g$-factor of $2.01 .^{51,52}$ Hence, we conclude that the measured Raman signals have to be attributed to spin flips of the $\mathrm{Mn}^{2+}$ ions interacting with the photogenerated exciton. We measured the spectral dependence of the Raman signal intensities. The maximal signal is reached when the laser is in resonance with the exciton. This shows that the exciton serves as an intermediate scattering state, which resonantly enhances the Raman cross-section.

The Raman signal is detected in all four combinations of circular polarizations of excitation and detection. The relative intensities $\left(\mathrm{I}^{++} / \mathrm{I}^{+-} / \mathrm{I}^{-+} / \mathrm{I}^{--}\right)$of the $\mathrm{Mn}^{2+}$ SFRS lines depend strongly on the $\mathrm{Mn}^{2+}$ concentration. Here $I^{i j}$ means $\sigma^{i}$-polarized excitation and $\sigma^{j}$-polarized detection. These intensities on the Stokes side for $n=1$ at $B=4 \mathrm{~T}$ are given by $(1 / 0.95 / 0.99 / 0.95)$ for CdSe $/ \mathrm{Cd}_{0.991} \mathrm{Mn}_{0.009} \mathrm{~S}$ (sample \#1), (1/0.75/0.84/0.62) for $\mathrm{CdSe} / \mathrm{Cd}_{0.99} \mathrm{Mn}_{0.01} \mathrm{~S}$ (sample \#2), and $(1 / 0.33 / 0.42 / 0.18)$ for $\mathrm{CdSe} / \mathrm{Cd}_{0.971} \mathrm{Mn}_{0.029} \mathrm{~S}$ (sample \#3). One can conclude that the optical selection rules become more distinct with increasing $\mathrm{Mn}^{2+}$ concentration and the $\mathrm{Mn}^{2+}$ lines become more dominant for $\sigma^{+}$polarized excitation and detection.

The observation of multiple spin flip $\mathrm{Mn}^{2+}$ lines was reported also for (Cd,Mn)Te-based quantum wells, where up to 15 spinflip lines were observed. ${ }^{54} \mathrm{~A}$ mechanism for these flips was suggested in ref 54 and a corresponding model description was developed in refs 34,59 . The key point of this model is the anisotropic spin of the heavy-hole in a two-dimensional 
nanostructure. In an external magnetic field, the $\mathrm{Mn}^{2+}$ spins are polarized along the field direction. When the spin of the photogenerated hole is not parallel to the magnetic field (for simplicity the case when it is perpendicular to the field can be considered), the $\mathrm{Mn}^{2+}$ spins are influenced by the external field $\mathbf{B}$ and by the hole exchange field $\mathbf{B}_{\text {exch }}$. The total magnetic moment of all $\mathrm{Mn}^{2+}$ spins within the hole localization volume, $I_{\mathrm{Mn}}$, precesses about the total field $\mathbf{B}+\mathbf{B}_{\text {exch. }}$. When the exciton recombines, i.e. scatters, the projection of $I_{\mathrm{Mn}}$ on $\mathbf{B}$ differs from the initial value by a multiple of the energy of the $n=1 \mathrm{Mn}^{2+}$ spin-flip. Note that the electron with an isotropic spin does not support this mechanism. Therefore, we can conclude that in the studied DMS NPLs the holes have sufficient wave function overlap with the shell $\mathrm{Mn}^{2+}$ spins for providing multiple SFRS. This is in line with our conclusions from the DCP data analysis.

NPLs have a close analogy to quantum wells and the model approach suggested for quantum wells can be directly applied also here. The only specifics, which need to be accounted for, are the varying orientations of the NPLs in an ensemble measurement. As we have noticed above, the condition for observation of multiple $\mathrm{Mn}^{2+}$-flips is the noncollinearity of $\mathbf{B}$ and $\mathbf{B}_{\text {exch. }}$. This means that in quantum wells the effect should be absent in the Faraday geometry, where the magnetic field is parallel to the structure growth axis. In DMS NPLs we observe the same amount of higher order spin scattering resonances in Faraday and Voigt geometry. We explain this result by the random orientation of the NPLs in the studied ensembles, leading to the situation that in any configuration a fraction of NPLs fulfills the condition for multiple $\mathrm{Mn}^{2+}$-flips. It is worthwhile to note that the model developed for the exciton, namely for the hole in the exciton, can be readily applied for the negatively charged exciton, as the hole spin acts similar on $\mathrm{Mn}^{2+}$ ions in both cases.

\section{CONCLUSIONS}

In conclusion, we have demonstrated the exchange interaction of excitons (trions) with the $\mathrm{Mn}^{2+}$ ions in $\mathrm{CdSe} / \mathrm{Cd}_{1-x} \mathrm{Mn}_{x} \mathrm{~S}$ core/shell nanoplatelets by means of polarized PL, optically detected magnetic resonance and spin-flip Raman scattering. One can conclude that these structures can be studied in detail by these experimental approaches that were established for diluted magnetic semiconductors. In particular, assessment of the dynamics for spin-lattice relaxation gives accurate estimates for the $\mathrm{Mn}^{2+}$ ion concentration. Our studies may help to functionalize colloidal DMS nanocrystals as magnetic or magneto-optical markers.

\section{METHODS}

Magneto-optical measurements. The NPLs were dropcasted on a substrate and mounted in a titanium sample holder on top of a threeaxis piezo-positioner and placed in the variable temperature insert $(4.2-70 \mathrm{~K})$ of a liquid helium bath cryostat equipped with a superconducting solenoid (magnetic fields up to $15 \mathrm{~T}$ ). The measurements were performed in the Faraday geometry (light excitation and detection parallel to the magnetic field direction). The PL was excited with a diode laser (photon energy $3.06 \mathrm{eV}$, wavelength $405 \mathrm{~nm}$ ) in continuous-wave or pulsed mode (pulse duration $50 \mathrm{ps,}$ pulse repetition rate $500 \mathrm{kHz}$ ) with a weak average excitation density of $0.5 \mathrm{~mW} / \mathrm{cm}^{2}$. The PL was dispersed with a $0.5-\mathrm{m}$ spectrometer and detected either by a liquid-nitrogen-cooled charge-coupled-device (CCD) camera or a $\mathrm{Si}$ avalanche photodiode connected to a conventional time-correlated single-photon counting system. The instrumental response time was about 200 ps. The PL circular polarization degree was analyzed by a combination of a quarter-wave plate and a linear polarizer.
Spin-flip Raman scattering. The samples were mounted strain free inside the variable temperature insert of a magnet cryostat, which provided magnetic fields up to $10 \mathrm{~T}$. The temperature was set to $1.6 \mathrm{~K}$. The backscattering experiments were performed in Faraday geometry $\left(\theta=0^{\circ}\right)$ or in tilted geometries up to $\theta=90^{\circ}$, corresponding to the Voigt geometry, where the magnetic field and the normal to the sample substrate enclose the angle $\theta$. The NPLs were excited by a single frequency dye laser (Matisse DS), whose actual wavelength was measured and monitored by a fiber-coupled wavelength-meter device. The laser power was stabilized by a liquid-crystal variable attenuator. Unless specified otherwise, the power was set to about $0.2 \mathrm{~W} / \mathrm{cm}^{2}$ at the sample surface. In order to ensure a stable detection position on the sample surface, each sample was covered by a mask having a hole of 1 $\mathrm{mm}$ diameter and the central part with $100 \times 100 \mu \mathrm{m}^{2}$ size of the illuminated sample area was selected by a cross slit. The NPL emission was spectrally dispersed by a double monochromator (U1000) equipped with a Peltier-cooled GaAs photomultiplier. The SFRS spectra were measured in close vicinity of the laser line with photon energy $E_{\text {exc }}$. The spin-flip signals were shifted from the laser energy by the Zeeman splitting of the involved spin state, either to lower (Stokes shift, $\left.E_{\text {exc }}-|g| \mu_{\mathrm{B}} B\right)$ or higher energies (anti-Stokes shift, $E_{\text {exc }}+|g| \mu_{\mathrm{B}} B$ ).

Optically detected magnetic resonance. The ODMR technique used in this study was described in detail in ref 49. The ODMR spectrometer consisted of a $60 \mathrm{GHz}$ all-solid-state microwave oscillator (photon energy of $0.248 \mathrm{meV}$ ) with a tuning range from 59.05 to 60.55 $\mathrm{GHz}$ and an output power of up to $100 \mathrm{~mW}$. The output power of the oscillator could be varied by up to $40 \mathrm{~dB}$ attenuation level. The oscillator could operate either in continuous-wave mode or in a periodically pulsed mode with an on-off transition time of about $3 \mathrm{~ns}$ at more than $60 \mathrm{~dB}$ dumping level. The sample was mounted in a cylindrical $\mathrm{H}_{011}$ microwave cavity with a low $\mathrm{Q}$ factor of about 600 . The cavity had two orthogonal pairs of apertures with a conic cross-section for sample illumination and collecting the sample emission. The cavity was placed in the variable temperature insert of a magnet cryostat, the measurements were performed at $T=1.8 \mathrm{~K}$. The sample in the cavity was excited by a $405 \mathrm{~nm}\left(E_{\text {exc }}=3.06 \mathrm{eV}\right)$ semiconductor laser in $\mathrm{cw}$ mode with $0.5 \mathrm{~mW}$ power, focused into a spot with a diameter of 400 $\mu \mathrm{m}$. The PL was collected in backscattering geometry and detected with a $0.5-\mathrm{m}$ grating monochromator and a CCD camera. Magnetic fields up to $7 \mathrm{~T}$ were applied in the Faraday geometry. For time-resolved ODMR measurements a photon counter based on an avalanche photodiode was used, the temporal resolution was $30 \mathrm{~ns}$.

\section{ASSOCIATED CONTENT}

\section{Supporting Information}

The Supporting Information is available free of charge at https://pubs.acs.org/doi/10.1021/acsnano.0c04048.

Additional information on synthesis details, XRD data, spectrally resolved PL decays, exciton bright-dark splitting, calculation of the band structure in core/shell NPLs, modeling of the exciton exchange interaction with $\mathrm{Mn}^{2+}$ ions, Zeeman splitting and polarization degree for the bright and dark excitons and negative trions (PDF)

\section{AUTHOR INFORMATION}

\section{Corresponding Authors}

Elena V. Shornikova - Experimentelle Physik 2, Technische

Universität Dortmund, 44227 Dortmund, Germany;

(1) orcid.org/0000-0002-6866-9013;

Email: elena.shornikova@tu-dortmund.de

Dmitri R. Yakovlev - Experimentelle Physik 2, Technische Universität Dortmund, 44227 Dortmund, Germany; Ioffe Institute, Russian Academy of Sciences, 194021 St. Petersburg, Russia; 이이이.org/0000-0001-7349-2745;

Email: dmitri.yakovlev@tu-dortmund.de 


\section{Authors}

Danil O. Tolmachev - Experimentelle Physik 2, Technische Universität Dortmund, 44227 Dortmund, Germany; Ioffe Institute, Russian Academy of Sciences, 194021 St. Petersburg, Russia; (1) orcid.org/0000-0002-7098-8515

Vitalii Yu. Ivanov - Institute of Physics, Polish Academy of Sciences, PL-02-668 Warsaw, Poland; 이이이.org/0000-00024651-8476

Ina V. Kalitukha - Ioffe Institute, Russian Academy of Sciences, 194021 St. Petersburg, Russia

Victor F. Sapega - Ioffe Institute, Russian Academy of Sciences, 194021 St. Petersburg, Russia

Dennis Kudlacik - Experimentelle Physik 2, Technische Universität Dortmund, 44227 Dortmund, Germany; (1) orcid.org/0000-0001-5473-8383

Yuri G. Kusrayev - Ioffe Institute, Russian Academy of Sciences, 194021 St. Petersburg, Russia

Aleksandr A. Golovatenko - Ioffe Institute, Russian Academy of Sciences, 194021 St. Petersburg, Russia; ㅇoㄷd.org/00000003-2248-3157

Sushant Shendre - LUMINOUS! Center of Excellence for Semiconductor Lighting and Displays, School of Electrical and Electronic Engineering, School of Physical and Materials Sciences, Nanyang Technological University, 639798, Singapore

Savas Delikanli - LUMINOUS! Center of Excellence for Semiconductor Lighting and Displays, School of Electrical and Electronic Engineering, School of Physical and Materials Sciences, Nanyang Technological University, 639798, Singapore; Department of Electrical and Electronics Engineering, Department of Physics, UNAM - Institute of Materials Science and Nanotechnology, Bilkent University, 06800 Ankara, Turkey; (i) orcid.org/0000-0002-0613-8014

Hilmi Volkan Demir - LUMINOUS! Center of Excellence for Semiconductor Lighting and Displays, School of Electrical and Electronic Engineering, School of Physical and Materials Sciences, Nanyang Technological University, 639798, Singapore; Department of Electrical and Electronics Engineering, Department of Physics, UNAM - Institute of Materials Science and Nanotechnology, Bilkent University, 06800 Ankara, Turkey; ○ orcid.org/0000-0003-1793-112X

Manfred Bayer - Experimentelle Physik 2, Technische Universität Dortmund, 44227 Dortmund, Germany; Ioffe Institute, Russian Academy of Sciences, 194021 St. Petersburg, Russia;

(1) orcid.org/0000-0002-0893-5949

Complete contact information is available at:

https://pubs.acs.org/10.1021/acsnano.0c04048

\section{Notes}

The authors declare no competing financial interest.

\section{ACKNOWLEDGMENTS}

The authors are thankful to A. V. Rodina for fruitful discussions. This work was supported by the Deutsche Forschungsgemeinschaft through the International Collaborative Research Center TRR 160 (Projects B1, B2, and C7) and by the Russian Foundation for Basic Research (Grant No. 19-52-12064 NNIOa). D.R.Y. acknowledges the partial support of the Russian Science Foundation (Project No. 20-42-01008). S.S., S.D. and H.V.D. acknowledge partial support from the Singapore National Research Foundation under NRF-NRFI2016-08. A.A.G. acknowledges support of the Grants Council of the President of the Russian Federation. V.Yu.I. acknowledges support of the Polish National Science Center (Grant No. 2018/ 30/M/ST3/00276). H.V.D. gratefully acknowledges support from TUBA. Yu.G.K. acknowledges the support of the Russian Science Foundation (Project No. 18-12-00352).

\section{REFERENCES}

(1) Efros, Al. L.; Rashba, E. I.; Rosen, M. Paramagnetic Ion-Doped Nanocrystal as a Voltage-Controlled Spin Filter. Phys. Rev. Lett. 2001, $87,206601$.

(2) Beaulac, R.; Archer, P. I.; Ochsenbein, S. T.; Gamelin, D. R. $\mathrm{Mn}^{2+}$ Doped CdSe Quantum Dots: New Inorganic Materials for SpinElectronics and Spin-Photonics. Adv. Funct. Mater. 2008, 18, 38733891.

(3) Muckel, F.; Barrows, C. J.; Graf, A.; Schmitz, A.; Erickson, C. S.; Gamelin, D. R.; Bacher, G. Current-Induced Magnetic Polarons in a Colloidal Quantum-Dot Device. Nano Lett. 2017, 17, 4768-4773.

(4) Moro, F.; Fielding, A. J.; Turyanska, L.; Patanè, A. Realization of Universal Quantum Gates with Spin-Qudits in Colloidal Quantum Dots. Adv. Quantum Technol. 2019, 2, 1900017.

(5) Furdyna, J. K. Diluted Magnetic Semiconductors. J. Appl. Phys. 1988, 64, R29-R64.

(6) Furdyna, J. K.; Kossut, J. In Diluted Magnetic Semiconductors; Furdyna, J. K., Kossut, J., Eds.; Semiconductors and Semimetals; Academic Press: London, 1988; Vol. 25.

(7) Gaj, J. A.; Kossut, J. In Introduction to the Physics of Diluted Magnetic Semiconductors; Kossut, J., Gaj, J. A., Eds.; Springer Series in Materials Science; Springer Verlag: Berlin, Heidelberg, 2010.

(8) Hoffman, D. M.; Meyer, B. K.; Ekimov, A. I.; Merkulov, I. A.; Efros, Al. L.; Rosen, M.; Couino, G.; Gacoin, T.; Boilot, J. P. Giant Internal Magnetic Fields in Mn Doped Nanocrystal Quantum Dots. Solid State Commun. 2000, 114, 547-550.

(9) Norris, D. J.; Yao, N.; Charnock, F. T.; Kennedy, T. A. HighQuality Manganese-Doped ZnSe Nanocrystals. Nano Lett. 2001, 1, 37 .

(10) Archer, P. I.; Santangelo, S. A.; Gamelin, D. R. Direct Observation of $\mathrm{sp}-\mathrm{d}$ Exchange Interactions in Colloidal $\mathrm{Mn}^{2+}$ - and $\mathrm{Co}^{2+}$-Doped CdSe Quantum Dots. Nano Lett. 2007, 7, 1037-1043.

(11) Bussian, D. A.; Crooker, S. A.; Yin, M.; Brynda, M.; Efros, Al. L.; Klimov, V. I. Tunable Magnetic Exchange Interactions in ManganeseDoped Inverted Core-Shell ZnSe-CdSe Nanocrystals. Nat. Mater. 2009, 8, 35-40.

(12) Pinchetti, V.; Di, Q.; Lorenzon, M.; Camellini, A.; Fasoli, M.; Zavelani-Rossi, M.; Meinardi, F.; Zhang, J.; Crooker, S. A.; Brovelli, S. Excitonic Pathway to Photoinduced Magnetism in Colloidal Nanocrystals with Nonmagnetic Dopants. Nat. Nanotechnol. 2018, 13, 145151.

(13) Beaulac, R.; Archer, P. I.; Liu, X.; Lee, S.; Salley, G. M.; Dobrowolska, M.; Furdyna, J. K.; Gamelin, D. R. Spin-Polarizable Excitonic Luminescence in Colloidal $\mathrm{Mn}^{2+}$-Doped CdSe Quantum Dots. Nano Lett. 2008, 8, 1197-1201.

(14) Long, G.; Barman, B.; Delikanli, S.; Tsung Tsai, Y.; Zhang, P.; Petrou, A.; Zeng, H. Carrier-Dopant Exchange Interactions in MnDoped PbS Colloidal Quantum Dots. Appl. Phys. Lett. 2012, 101, No. 062410.

(15) Turyanska, L.; Hill, R. J. A.; Makarovsky, O.; Moro, F.; Knott, A. N.; Larkin, O. J.; Patanè, A.; Meaney, A.; Christianen, P. C. M.; Fay, M. W.; Curry, R. J. Tuneable Paramagnetic Susceptibility and Exciton gFactor in Mn-Doped PbS Colloidal Nanocrystals. Nanoscale 2014, 6, 8919-8925.

(16) Delikanli, S.; Akgul, M. Z.; Murphy, J. R.; Barman, B.; Tsai, Y.; Scrace, T.; Zhang, P.; Bozok, B.; Hernández-Martínez, P. L.; Christodoulides, J.; Cartwright, A. N.; Petrou, A.; Demir, H. V. $\mathrm{Mn}^{2+}$-Doped CdSe/CdS Core/Multishell Colloidal Quantum Wells Enabling Tunable Carrier-Dopant Exchange Interactions. ACS Nano 2015, 9, 12473-12479.

(17) Murphy, J. R.; Delikanli, S.; Scrace, T.; Zhang, P.; Norden, T.; Thomay, T.; Cartwright, A. N.; Demir, H. V.; Petrou, A. Time-Resolved 
Photoluminescence Study of CdSe/CdMnS/CdS Core/Multi-Shell Nanoplatelets. Appl. Phys. Lett. 2016, 108, 242406.

(18) Najafi, A.; Tarasek, S.; Delikanli, S.; Zhang, P.; Norden, T.; Shendre, S.; Sharma, M.; Bhattacharya, A.; Taghipour, N.; Pientka, J.; Demir, H. V.; Petrou, A.; Thomay, T. CdSe/CdMnS Nanoplatelets with Bilayer Core and Magnetically Doped Shell Exhibit Switchable Excitonic Circular Polarization: Implications for Lasers and LightEmitting Diodes. ACS Appl. Nano Mater. 2020, 3, 3151-3156.

(19) Strassberg, R.; Delikanli, S.; Barak, Y.; Dehnel, J.; Kostadinov, A.; Maikov, G.; Hernandez-Martinez, P. L.; Sharma, M.; Demir, H. V.; Lifshitz, E. Persuasive Evidence for Electron-Nuclear Coupling in Diluted Magnetic Colloidal Nanoplatelets Using Optically Detected Magnetic Resonance Spectroscopy. J. Phys. Chem. Lett. 2019, 10, 4437-4447.

(20) Beaulac, R.; Schneider, L.; Archer, P. I.; Bacher, G.; Gamelin, D. R. Light-Induced Spontaneous Magnetization in Doped Colloidal Quantum Dots. Science 2009, 325, 973-976.

(21) Rice, W. D.; Liu, W.; Pinchetti, V.; Yakovlev, D. R.; Klimov, V. I.; Crooker, S. A. Direct Measurements of Magnetic Polarons in $\mathrm{Cd}_{1-x} \mathrm{Mn}_{x}$ Se Nanocrystals from Resonant Photoluminescence. Nano Lett. 2017, 17, 3068-3075.

(22) Lorenz, S.; Erickson, C. S.; Riesner, M.; Gamelin, D. R.; Fainblat, R.; Bacher, G. Directed Exciton Magnetic Polaron Formation in a Single Colloidal $\mathrm{Mn}^{2+}$ : CdSe/CdS Quantum Dot. Nano Lett. 2020, 20, 1896-1906.

(23) Rice, W. D.; Liu, W.; Baker, T. A.; Sinitsyn, N. A.; Klimov, V. I.; Crooker, S. A. Revealing Giant Internal Magnetic Fields Due to Spin Fluctuations in Magnetically Doped Colloidal Nanocrystals. Nat. Nanotechnol. 2016, 11, 137-142.

(24) Ithurria, S.; Dubertret, B. Quasi 2D Colloidal CdSe Platelets with Thicknesses Controlled at the Atomic Level. J. Am. Chem. Soc. 2008, 130, 16504-16505.

(25) Furdyna, J. K.; Lee, S.; Dobrowolska, M.; Wojtowicz, T.; Liu, X. Band-Offset Engineering in Magnetic/Non-Magnetic Semiconductor Quantum Structures. In Introduction to the Physics of Diluted Magnetic Semiconductors; Kossut, J., Gaj, J. A., Eds.; Springer Series in Materials Science; Springer Verlag: Berlin, Heidelberg, 2010; pp 103-160.

(26) Muckel, F.; Delikanli, S.; Hernandez-Martinez, P. L.; Priesner, T.; Lorenz, S.; Ackermann, J.; Sharma, M.; Demir, H. V.; Bacher, G. sp-d Exchange Interactions in Wave Function Engineered Colloidal CdSe/ Mn:CdS Hetero-Nanoplatelets. Nano Lett. 2018, 18, 2047-2053.

(27) Zhang, P.; Norden, T.; Pientka, J. M.; Oszwałdowski, R.; Najafi, A.; Barman, B.; Tsai, Y.; Fan, W.-C.; Chou, Wu-C.; Han, J. E.; Žutić, I.; McCombe, B. D.; Petrou, A. Optical Control of Hole Wavefunction in Type-II Magnetic Quantum Dot Structures. J. Phys. Chem. C 2019, 123, 25934-25940.

(28) Davis, A. H.; Hofman, E.; Chen, K.; Li, Z.-J.; Khammang, A.; Zamani, H.; Franck, J. M.; Maye, M. M.; Meulenberg, R. W.; Zheng, W. Exciton Energy Shifts and Tunable Dopant Emission in ManganeseDoped Two-Dimensional CdS/ZnS Core/Shell Nanoplatelets. Chem. Mater. 2019, 31, 2516-2523.

(29) Delikanli, S.; Yu, G.; Yeltik, A.; Bose, S.; Erdem, T.; Yu, J.; Erdem, O.; Sharma, M.; Sharma, V. K.; Quliyeva, U.; Shendre, S.; Dang, C.; Zhang, D. H.; Sum, T. C.; Fan, W.; Demir, H. V. Ultrathin Highly Luminescent Two-Monolayer Colloidal CdSe Nanoplatelets. Adv. Funct. Mater. 2019, 29, 1901028.

(30) Shendre, S.; Delikanli, S.; Li, M.; Dede, D.; Pan, Z.; Ha, S. T.; Fu, Y. H.; Hernández Martínez, P. L.; Yu, J.; Erdem, O.; Kuznetsov, A. I.; Dang, C.; Sum, T. C.; Demir, H. V. Ultrahigh-Efficiency Aqueous Flat Nanocrystals of CdSe/CdS@ $\mathrm{Cd}_{1-x} \mathrm{Zn}_{x} \mathrm{~S}$ Colloidal Core/Crown@ Alloyed-Shell Quantum Wells. Nanoscale 2019, 11, 301-310.

(31) Adachi, S. Handbook on Physical Properties of Semiconductors; Springer US: Boston, MA, 2004.

(32) Javaux, C.; Mahler, B.; Dubertret, B.; Shabaev, A.; Rodina, A. V.; Efros, Al. L.; Yakovlev, D. R.; Liu, F.; Bayer, M.; Camps, G.; Biadala, L.; Buil, S.; Quelin, X.; Hermier, J.-P. Thermal Activation of Non-Radiative Auger Recombination in Charged Colloidal Nanocrystals. Nat. Nanotechnol. 2013, 8, 206-212.
(33) Merkulov, I. A.; Yakovlev, D. R.; Keller, A.; Ossau, W.; Geurts, J.; Waag, A.; Landwehr, G.; Karczewski, G.; Wojtowicz, T.; Kossut, J. Kinetic Exchange between the Conduction Band Electrons and Magnetic Ions in Quantum-Confined Structures. Phys. Rev. Lett. 1999, 83, 1431-1434.

(34) Merkulov, I. A., Rodina, A. V. Exchange Interaction between Carriers and Magnetic Ions in Quantum Size Heterostructures. In Introduction to the Physics of Diluted Magnetic Semiconductors; Kossut, J., Gaj, J. A., Eds.; Springer Series in Materials Science; Springer Verlag: Berlin, Heidelberg, 2010; pp 65-101.

(35) Yakovlev, D. R., Merkulov, I. A. Spin and Energy Transfer between Carriers, Magnetic Ions, and Lattice. In Introduction to the Physics of Diluted Magnetic Semiconductors; Kossut, J., Gaj, J. A., Eds.; Springer Series in Materials Science; Springer Verlag: Berlin, Heidelberg, 2010; pp 263-303.

(36) Kneip, M. K.; Yakovlev, D. R.; Bayer, M.; Karczewski, G.; Wojtowicz, T.; Kossut, J. Engineering of Spin-Lattice Relaxation Dynamics by Digital Growth of Diluted Magnetic Semiconductor CdMnTe. Appl. Phys. Lett. 2006, 88, 152105.

(37) Shornikova, E. V.; Biadala, L.; Yakovlev, D. R.; Feng, D.; Sapega, V. F.; Flipo, N.; Golovatenko, A. A.; Semina, M. A.; Rodina, A. V.; Mitioglu, A. A.; Ballottin, M. V.; Christianen, P. C. M.; Kusrayev, Yu. G.; Nasilowski, M.; Dubertret, B.; Bayer, M. Electron and Hole gFactors and Spin Dynamics of Negatively Charged Excitons in CdSe/ CdS Colloidal Nanoplatelets with Thick Shells. Nano Lett. 2018, 18, 373-380.

(38) Müller, E.; Gebhardt, W. Position and Lifetime of Photoluminescence in $\mathrm{Cd}_{1-x} \mathrm{Mn}_{x} \mathrm{Te}$ and $\mathrm{Zn}_{1-x} \mathrm{Mn}_{x} \mathrm{Te}$. Exchange Dependent Effects. Phys. Status Solidi B 1986, 137, 259-267.

(39) Schenk, H.; Wolf, M.; Mackh, G.; Zehnder, U.; Ossau, W.; Waag, A.; Landwehr, G. Influence of the Negative Thermal-Expansion Coefficient on the Luminescence Properties of (CdMnMg)Te. J. Appl. Phys. 1996, 79, 8704-8711.

(40) Beaulac, R.; Archer, P. I.; van Rijssel, J.; Meijerink, A.; Gamelin, D. R. Exciton Storage by $\mathrm{Mn}^{2+}$ in Colloidal $\mathrm{Mn}^{2+}$-Doped CdSe Quantum Dots. Nano Lett. 2008, 8, 2949-2953.

(41) Shornikova, E. V.; Biadala, L.; Yakovlev, D. R.; Sapega, V. F.; Kusrayev, Y. G.; Mitioglu, A. A.; Ballottin, M. V.; Christianen, P. C. M.; Belykh, V. V.; Kochiev, M. V.; Sibeldin, N. N.; Golovatenko, A. A.; Rodina, A. V.; Gippius, N. A.; Kuntzmann, A.; Jiang, Ye; Nasilowski, M.; Dubertret, B.; Bayer, M. Addressing the Exciton Fine Structure in Colloidal Nanocrystals: the Case of CdSe Nanoplatelets. Nanoscale 2018, 10, 646-656.

(42) Shornikova, E. V.; Yakovlev, D. R.; Biadala, L.; Crooker, S. A.; Belykh, V. V.; Kochiev, M. V.; Kuntzmann, A.; Nasilowski, M.; Dubertret, B.; Bayer, M. Negatively Charged Excitons in CdSe Nanoplatelets. Nano Lett. 2020, 20, 1370-1377.

(43) Furis, M.; Hollingsworth, J. A.; Klimov, V. I.; Crooker, S. A. Time- and Polarization-Resolved Optical Spectroscopy of Colloidal CdSe Nanocrystal Quantum Dots in High Magnetic Fields. J. Phys. Chem. B 2005, 109, 15332-15338.

(44) Liu, F.; Rodina, A. V.; Yakovlev, D. R.; Golovatenko, A. A.; Greilich, A.; Vakhtin, E. D.; Susha, A.; Rogach, A. L.; Kusrayev, Yu. G.; Bayer, M. Förster Energy Transfer of Dark Excitons Enhanced by a Magnetic Field in an Ensemble of CdTe Colloidal Nanocrystals. Phys. Rev. B: Condens. Matter Mater. Phys. 2015, 92, 125403.

(45) Liu, F.; Biadala, L.; Rodina, A. V.; Yakovlev, D. R.; Dunker, D.; Javaux, C.; Hermier, J.-P.; Efros, Al. L.; Dubertret, B.; Bayer, M. Spin Dynamics of Negatively Charged Excitons in CdSe/CdS Colloidal Nanocrystals. Phys. Rev. B: Condens. Matter Mater. Phys. 2013, 88, No. 035302.

(46) Shornikova, E. V.; Golovatenko, A. A.; Yakovlev, D. R.; Rodina, A. V.; Biadala, L.; Qiang, G.; Kuntzmann, A.; Nasilowski, M.; Dubertret, B.; Polovitsyn, A.; Moreels, I.; Bayer, M. Surface Spin Magnetism Controls the Polarized Exciton Emission from CdSe Nanoplatelets. Nat. Nanotechnol. 2020, 15, 277-282.

(47) Efros, Al. L. Fine Structure and Polarization Properties of BandEdge Excitons in Semiconductor Nanocrystals. In Semiconductor and 
Metal Nanocrystals: Synthesis and Electronic and Optical Properties;

Klimov, V. I., Ed.; Dekker: New York, 2003; pp 103-141.

(48) Brumberg, A.; Harvey, S. M.; Philbin, J. P.; Diroll, B. T.; Lee, B.;

Crooker, S. A.; Wasielewski, M. R.; Rabani, E.; Schaller, R. D.

Determination of the In-Plane Exciton Radius in 2D CdSe Nanoplatelets via Magneto-Optical Spectroscopy. ACS Nano 2019, 13, $8589-8596$.

(49) Ivanov, V. Yu; Godlewski, M.; Yakovlev, D. R.; Ryabchenko, S. M.; Karczewski, G.; Waag, A. Time-Resolved Optically-Detected Magnetic Resonance of II-VI Diluted Magnetic-Semiconductor

Heterostructures. Phys. Status Solidi A 2007, 204, 174-178.

(50) Ivanov, V. Yu; Godlewski, M.; Yakovlev, D. R.; Kneip, M. K.; Bayer, M.; Ryabchenko, S. M.; Waag, A. Optically Detected Magnetic Resonance in $(\mathrm{Zn}, \mathrm{Mn}) \mathrm{Se} /(\mathrm{Zn}, \mathrm{Be}) \mathrm{Se}$ Quantum Wells. Phys. Rev. B: Condens. Matter Mater. Phys. 2008, 78, No. 085322.

(51) Matarrse, L.M.; Kikuchi, C. Paramagnetic Resonance Absorption of $\mathrm{Mn}^{+2}$ in Single Crystals of Zincblende. J. Phys. Chem. Solids 1956, 1, $117-127$.

(52) Lambe, J.; Kikuchi, C. Paramagnetic Resonance of CdTe:Mn and CdS:Mn. Phys. Rev. 1960, 119, 1256-1260.

(53) Heiman, D.; Wolff, P. A.; Warnock, J. Spin-Flip Raman Scattering, Bound Magnetic Polaron, and Fluctuations in (Cd,Mn)Se. Phys. Rev. B: Condens. Matter Mater. Phys. 1983, 27, 4848-4860.

(54) Stühler, J.; Schaack, G.; Dahl, M.; Waag, A.; Landwehr, G.; Kavokin, K. V.; Merkulov, I. A. Multiple $\mathrm{Mn}^{2+}$-Spin-Flip Raman Scattering at High Fields via Magnetic Polaron States in Semimagnetic Quantum Wells. Phys. Rev. Lett. 1995, 74, 2567-2570.

(55) Bao, J. M.; Bragas, A. V.; Furdyna, J. K.; Merlin, R. Control of Spin Dynamics with Laser Pulses: Generation of Entangled States of Donor-Bound Electrons in a $\mathrm{Cd}_{1-x} \mathrm{Mn}_{x}$ Te Quantum Well. Phys. Rev. B: Condens. Matter Mater. Phys. 2005, 71, No. 045314.

(56) Smith, L. C.; Davies, J. J.; Wolverson, D.; Lentze, M.; Geurts, J.; Wojtowicz, T.; Karczewski, G. Dependence of Multiple $\mathrm{Mn}^{2+}$ Spin-Flip Raman Scattering in Quantum Wells on the Magnetic Field Direction. Phys. Rev. B: Condens. Matter Mater. Phys. 2008, 77, 115341.

(57) Kudlacik, D.; Sapega, V. F.; Yakovlev, D. R.; Kalitukha, I. V.; Shornikova, E. V.; Rodina, A. V.; Ivchenko, E. L.; Dimitriev, G. S.; Nasilowski, M.; Dubertret, B.; Bayer, M. Single and Double Electron Spin-Flip Raman Scattering in CdSe Colloidal Nanoplatelets. Nano Lett. 2020, 20, 517-525.

(58) Feng, D.; Yakovlev, D. R.; Dubertret, B.; Bayer, M. Charge Separation Dynamics in CdSe/CdS Core/Shell Nanoplatelets Addressed by Coherent Electron Spin Precession. ACS Nano 2020, 14, 7237-7244.

(59) Kavokin, K. V.; Merkulov, I. A. Multispin Raman Paramagnetic Resonance: Quantum Dynamics of Classically Large Angular Momenta. Phys. Rev. B: Condens. Matter Mater. Phys. 1997, 55, R7371-R7374. 